\title{
GOE-GUE-Poisson transitions in the nearest neighbor spacing distribution of magnetoexcitons
}

\author{
Frank Schweiner, Jörg Main, and Günter Wunner \\ Institut für Theoretische Physik 1, Universität Stuttgart, 70550 Stuttgart, Germany
}

(Dated: July 14, 2018)

\begin{abstract}
Recent investigations on the Hamiltonian of excitons by F. Schweiner et al. [Phys. Rev. Lett. 118, 046401 (2017)] revealed that the combined presence of a cubic band structure and external fields breaks all antiunitary symmetries. The nearest neighbor spacing distribution of magnetoexcitons can exhibit Poissonian statistics, the statistics of a Gaussian orthogonal ensemble (GOE) or a Gaussian unitary ensemble (GUE) depending on the system parameters. Hence, magnetoexcitons are an ideal system to investigate the transitions between these statistics. Here we investigate the transitions between GOE and GUE statistics and between Poissonian and GUE statistics by changing the angle of the magnetic field with respect to the crystal lattice and by changing the scaled energy known from the hydrogen atom in external fields. Comparing our results with analytical formulae for these transitions derived with random matrix theory, we obtain a very good agreement and thus confirm the Wigner surmise for the exciton system.
\end{abstract}

PACS numbers: 05.30.Ch, 05.45.Mt, 71.35.-y, 61.50.-f

\section{INTRODUCTION}

Ever since the Bohigas-Giannoni-Schmit conjecture [1, which stated that these quantum systems can be described by random matrix theory [2, 3], it has been shown that irregular classical behavior manifests itself in statistical quantities of the corresponding quantum system 4 . In random matrix theory the Hamiltonian of a system is replaced by a random matrix with appropriate symmetries to study the statistical properties of its eigenvalue spectrum [5]; so only universal quantities of a system are considered and detailed dynamical properties are irrelevant. Even though Hamiltonians of dynamical systems are not random in most cases, it is already understood that spectral fluctuations for nonrandom and random Hamiltonians are equivalent [6-8].

All systems with a Hamiltonian leading to global chaos in the classical dynamics can be assigned to one of three universality classes: the orthogonal, the unitary or the symplectic universality class [7. To which of these universality classes a given system belongs is determined by the remaining symmetries in the system. Most of the physical systems still have time-reversal or at least one remaining antiunitary symmetry and thus show the statistics of a Gaussion orthogonal ensemble (GOE). Some examples of these systems are nuclei in external magnetic fields 9 -12, microwave billards 13 15, molecular spectra [16, impurities [17, and quantum wells [18. Atoms in constant external fields, in particular, are among the most important physical systems belonging to the orthogonal universality class [19 21]. They are ideal systems to investigate the emergence of quantum chaos both in highprecision experimental measurements and precise quantal calculations, possible because of the availability of the analytically known Hamiltonian (see Refs. 4, 22, and further references therein). Hence, they are a perfectly suitable physical system to study the transition from the Poissonian level statistics, which describes the classically integrable case in the absence of the fields [7, 23, to GOE statistics 21, where the breaking of symmetries due to the external fields leads to a correlation of levels and hence to a strong suppression of crossings [7.

As regards the other universality classes, examples are much rarer since systems without any antiunitary symmetry [Gaussian unitary ensemble (GUE)] or systems with time-reversal invariance possessing Kramer's degeneracy but no geometric symmetry at all [Gaussian symplectic ensemble (GSE)] have to be found [7. Until now GUE statistics was observable in rather exotic systems such as microwave cavities with ferrite strips 24, atoms in a static electric field and a resonant microwave field of elliptical polarization [25, a kicked rotor or a kicked top [6, 26, 27, the metal-insulator transition in the Anderson model of disordered systems [28, which can be compared to the Brownian motion model 29], or for billards in microwave resonators [30, and in graphene quantum dots 31. Since random matrix theory has already been extended to describe also transitions between the different statistics with analytical functions [5, it is highly desirable to study these transitions theoretically and experimentally. However, due to the small number of physical systems showing GUE statistics, there are only few examples, where transitions from Poissonian to GUE statistics or from GOE to GUE statistics in dependence of a parameter of the system could be studied [6, 2628, 32. Often only mathematical models with specifically designed Hamiltonians are introduced to investigate these transitions [5].

In this paper we will investigate these transitions in magnetoexcitons. Excitons are the fundamental optical excitations in the visible or ultraviolet spectrum of a semiconductor and consist of an electron in the conduction band and a positively charged hole in the valence band. As the interaction between both quasi particles can be described by a screened Coulomb interaction, excitons are often regarded as the hydrogen analog 
of the solid state. Only three years ago T. Kazimierczuk et al 33] observed in a remarkable high-resolution absorption experiment an almost perfect hydrogen-like absorption series for the yellow exciton in cuprous oxide $\left(\mathrm{Cu}_{2} \mathrm{O}\right)$ up to a principal quantum number of $n=25$. This experiment has opened the field of research of giant Rydberg excitons, and has stimulated a large number of experimental and theoretical investigations [33 49].

Very recently, we have shown that the Hamiltonian of magnetoexcitons in cubic semiconductors breaks all antiunitary symmetries [36. This is the first evidence for a spatially homogeneous system breaking all antiunitary symmetries.

Since in many cases excitons are treated theoretically via a hydrogen-like Hamiltonian, the appearance of GUE statistics seems surprising as the hydrogen atom in external fields still shows one antiunitary symmetry. However, it is well known that the hydrogen-like model of excitons is often too simple to account for the huge number of effects in the solid (see, e.g., Refs. [37, 40, 42, 50,54 and further references therein). M. Aßmann et al. 34, 35. attributed the appearance of GUE statistics in a recent experiment with magnetoexcitons in $\mathrm{Cu}_{2} \mathrm{O}$ to the interaction of excitons with phonons.

However, we have shown that it is indispensable to account for the complete valence band structure to describe the spectra of excitons in magnetic fields in a theoretically correct way [43]. Without the complete band structure the striking experimental finding of a dependence of the magnetoexciton spectra on the direction of the external magnetic field cannot be explained. It is indeed the simultaneous presence of the cubic band structure and external fields which breaks all antiunitary symmetries and leads to GUE statistics 36.

In this paper we investigate the symmetry breaking for excitons in semiconductors with a cubic band structure in dependence on system parameters such as the strength and the angle of the magnetic field or the scaled energy [4, 55. Since the eigenvalue spectrum of the magnetoexciton Hamiltonian shows Poissonian, GOE or GUE statistics depending on these parameters, it is an ideal system to investigate the transitions between GOE and GUE or Poisson and GUE statistics. To the best of our knowledge, there are only two more systems where both transitions have been studied, i.e., the kicked top 27 and the Anderson model 28. However, while the kicked top is a time-dependent system, which has to be treated within Floquet theory [6, 27], the Anderson model is rather a model system for a $d$-dimensional disordered lattice, where parameters such as the disorder and the hopping rate need to be adjusted 28]. Magnetoexcitons are a more realistic physical system allowing for a systematic investigation of transitions between different statistics. In particular, the parameters describing these transitions can be easily adjusted in experiments. Comparing our results with analytical functions from random matrix theory describing the transitions between the statistics [5, 6, we confirm the so-called Wigner surmise 56, which states that the NNS of large random matrices can be approximated by the NNS of $2 \times 2$ matrices of the same universality class [5].

The paper is organized as follows: In Sec. II we present the Hamiltonian of excitons in cubic semiconductors in an external magnetic field and introduce a complete basis to solve the corresponding Schrödinger equation. The methods of solving the Schrödinger equation for fixed values of the external field strenghts or for a constant scaled energy are discussed in Secs. IIA and IIB respectively. Having shown analytically that the presence of the cubic band structure and external fields breaks all antiunitary symmetries in Sec. III, we investigate the eigenvalue spectrum and the level spacing statistics numerically At first, we demonstrate the appearance of GOE or GUE statistics for specific directions of an external magnetic field in Sec. IV] The transitions between different level spacing statistics are then investigated in Secs. $\mathrm{VA}$ and $\mathrm{VB}$. Finally, we give a short summary and outlook in Sec.VI.

\section{HAMILTONIAN AND COMPLETE BASIS}

In this section we briefly discuss the Hamiltonian of excitons in direct semiconductors with a cubic valence band structure and show how to solve the corresponding Schrödinger equation in a complete basis. For more details see Refs. [2, 43, and further references therein.

When neglecting external fields at first, the Hamiltonian of excitons in direct semiconductors is given by [57]

$$
H=V\left(\boldsymbol{r}_{e}-\boldsymbol{r}_{h}\right)+H_{\mathrm{e}}\left(\boldsymbol{p}_{\mathrm{e}}\right)+H_{\mathrm{h}}\left(\boldsymbol{p}_{\mathrm{h}}\right) .
$$

The Coulomb interaction between the electron (e) and the hole $(\mathrm{h})$ is screened by the dielectric constant $\varepsilon$ :

$$
V\left(\boldsymbol{r}_{e}-\boldsymbol{r}_{h}\right)=-\frac{e^{2}}{4 \pi \varepsilon_{0} \varepsilon} \frac{1}{\left|\boldsymbol{r}_{e}-\boldsymbol{r}_{h}\right|} .
$$

Since the conduction band is often parabolic, the kinetic energy of the electron is similar to that of a free particle

$$
H_{\mathrm{e}}\left(\boldsymbol{p}_{\mathrm{e}}\right)=\frac{\boldsymbol{p}_{\mathrm{e}}^{2}}{2 m_{\mathrm{e}}} .
$$

However, the effective mass $m_{\mathrm{e}}$ of the electron in the semiconductor has to be used instead of the free electron mass $m_{0}$. As regards the valence bands, the situation is more complicated. In general, the uppermost valence band is threefold degenerate at the center of the Brillouin zone or the $\Gamma$ point and the kinetic energy of a hole within these valence bands is given by [41, 42]

$$
\begin{aligned}
H_{\mathrm{h}}\left(\boldsymbol{p}_{\mathrm{h}}\right) & =\left(1 / 2 \hbar^{2} m_{0}\right)\left\{\hbar^{2}\left(\gamma_{1}+4 \gamma_{2}\right) \boldsymbol{p}_{\mathrm{h}}^{2}\right. \\
& -6 \gamma_{2}\left(p_{\mathrm{h} 1}^{2} \boldsymbol{I}_{1}^{2}+\text { c.p. }\right) \\
& -12 \gamma_{3}\left(\left\{p_{\mathrm{h} 1}, p_{\mathrm{h} 2}\right\}\left\{\boldsymbol{I}_{1}, \boldsymbol{I}_{2}\right\}+\text { c.p. }\right)
\end{aligned}
$$


with $\boldsymbol{p}=\left(p_{1}, p_{2}, p_{3}\right),\{a, b\}=\frac{1}{2}(a b+b a)$ and c.p. denoting cyclic permutation. The three Luttinger parameters $\gamma_{i}$ describe the behavior and the anisotropic effective mass of the hole. The matrices $\boldsymbol{I}_{j}$ denote the three spin matrices of the quasispin $I=1$ which describes the threefold degenerate valence band [58. The components of these matrices $\boldsymbol{I}_{i}$ read [42, 58]

$$
I_{i, j k}=-i \hbar \varepsilon_{i j k}
$$

with the Levi-Civita symbol $\varepsilon_{i j k}$.

Note that the expression for $H_{\mathrm{h}}\left(\boldsymbol{p}_{\mathrm{h}}\right)$ can be separated in two parts having spherical and cubic symmetry, respectively [59]. The coefficients $\mu^{\prime}$ and $\delta^{\prime}$ of these parts can be expressed in terms of the three Luttinger parameters: $\mu^{\prime}=\left(6 \gamma_{3}+4 \gamma_{2}\right) / 5 \gamma_{1}^{\prime}$ and $\delta^{\prime}=\left(\gamma_{3}-\gamma_{2}\right) / \gamma_{1}^{\prime}$ with $\gamma_{1}^{\prime}=\gamma_{1}+m_{0} / m_{\mathrm{e}}$ [42, [53, 59]. The spin-orbit coupling $H_{\mathrm{so}}$, which generally enters the kinetic energy of the hole (4), is neglected here since it is spherically symmetric and therefore does not affect the symmetry properties of the exciton Hamiltonian.

When applying external fields, the corresponding Hamiltonian is obtained via the minimal substitution. After introducing relative and center of mass coordinates [60, 61] and setting the position and momentum of the center of mass to zero, the complete Hamiltonian of the relative motion reads [52, 60, 65.

$$
\begin{aligned}
H & =V(\boldsymbol{r})+e \Phi(\boldsymbol{r}) \\
& +H_{\mathrm{e}}(\boldsymbol{p}+e \boldsymbol{A}(\boldsymbol{r}))+H_{\mathrm{h}}(-\boldsymbol{p}+e \boldsymbol{A}(\boldsymbol{r}))
\end{aligned}
$$

with the relative coordinate $\boldsymbol{r}=\boldsymbol{r}_{\mathrm{e}}-\boldsymbol{r}_{\mathrm{h}}$ and the relative momentum $\boldsymbol{p}=\left(\boldsymbol{p}_{\mathrm{e}}-\boldsymbol{p}_{\mathrm{h}}\right) / 2$ of electron and hole. We use the vector potential $\boldsymbol{A}=(\boldsymbol{B} \times \boldsymbol{r}) / 2$ of a constant magnetic field $\boldsymbol{B}$ and the electrostatic potential $\Phi(\boldsymbol{r})=$ $-\boldsymbol{F} \cdot \boldsymbol{r}$ of a constant electric field $\boldsymbol{F}$.

As we will show in Sec. III, the symmetry breaking in the system depends on the orientation of the fields with respect to the crystal lattice. We will denote the orientation of $\boldsymbol{B}$ and $\boldsymbol{F}$ in spherical coordinates via

$$
\boldsymbol{B}(\varphi, \vartheta)=B\left(\begin{array}{c}
\cos \varphi \sin \vartheta \\
\sin \varphi \sin \vartheta \\
\cos \vartheta
\end{array}\right)
$$

and similar for $\boldsymbol{F}$ in what follows.

Before we solve the Schrödinger equation corresponding to the Hamiltonian (6), we rotate the coordinate system to make the quantization axis coincide with the direction of the magnetic field (see Appendix A) and then express the Hamiltonian (6) in terms of irreducible tensors [59, 65, 66]. We can then calculate a matrix representation of the Schrödinger equation using a complete basis.

Note that the Hamiltonian (6) is a model system for magnetoexcitons since we neglect the spin-orbit coupling between the quasi spin $I$ and the hole spin $S_{\mathrm{h}}$, which appears, e.g., in $\mathrm{Cu}_{2} \mathrm{O}$ 42, 43. Furthermore, we neglect an additional term in Eq. (6), which describes the energy of the electron and hole spin in the magnetic field but is invariant under the symmetry operations considered below. Therefore, we can disregard these spins in our basis. As regards the angular momentum part of the basis, we have to consider that the Hamiltonian (6) couples the angular momentum $L$ of the exciton and the quasi spin $I$. Hence, we introduce the total momentum $G=L+I$ with the $z$ component $M_{G}$. For the radial part of the exciton wave function we use the Coulomb-Sturmian functions of Ref. 67.

$$
U_{N L}(r)=N_{N L}(2 \rho)^{L} e^{-\rho} L_{N}^{2 L+1}(2 \rho)
$$

with $\rho=r / \alpha$, a normalization factor $N_{N L}$, the associated Laguerre polynomials $L_{n}^{m}(x)$ and an arbitrary scaling parameter $\alpha$. Note that we use the radial quantum number $N$, which is related to the principal quantum number $n$ via $n=N+L+1$. Finally, we make the following ansatz for the exciton wave function

$$
\begin{aligned}
|\Psi\rangle & =\sum_{N L G M_{G}} c_{N L G M_{G}}|\Pi\rangle, \\
|\Pi\rangle & =\left|N, L, I, G, M_{G}\right\rangle,
\end{aligned}
$$

with complex coefficients $c$.

The Schrödinger equation can now be solved for fixed values of the external field strengths or for a fixed value of the scaled energy known from atoms in external fields [55]. Both methods will be presented in the following

\section{A. Constant field strengths}

Inserting the ansatz (9) in the Schrödinger equation $H \Psi=E \Psi$ yields a matrix representation of the Schrödinger equation of the form [36]

$$
D c=E M c,
$$

where the external field strengths are assumed to be constant. The vector $\boldsymbol{c}$ contains the coefficients of the expansion (9). Since the functions $U_{N L}(r)$ actually depend on the coordinate $\rho=r / \alpha$, we substitute $r \rightarrow \rho \alpha$ in the Hamiltonian (6) and multiply the corresponding Schrödinger equation by $\alpha^{2}$. All matrix elements which enter the hermitian matrices $\boldsymbol{D}$ and $\boldsymbol{M}$ can be calculated similarly to the matrix elements given in Refs. 42, 43. The generalized eigenvalue problem $(10)$ is finally solved using an appropriate LAPACK routine 68.

Since in numerical calculations the basis cannot be infinitely large, the values of the quantum numbers are chosen in the following way: For each value of $n=$ $N+L+1 \leq n_{\max }$ we use

$$
\begin{aligned}
L & =0, \ldots, n-1, \\
G & =|L-1|, \ldots, \min \left(L+1, G_{\max }\right), \\
M_{G} & =-G, \ldots, G .
\end{aligned}
$$


The values $G_{\max }$ and $n_{\max }$ are chosen appropriately large so that as many eigenvalues as possible converge. Additionally, we can use the scaling parameter $\alpha$ to enhance convergence. In particular, if the eigenvalues of excitonic states with principal quantum number $n$ are to be be calculated, we can set $\alpha=n \gamma_{1}^{\prime} \varepsilon a_{0}$ according to Ref. [67, where $a_{0}$ denotes the Bohr radius.

Note that without an external electric field, parity is a good quantum number and the operators in the Schrödinger equation couple only basis states with even or with odd values of $L$. In this case we consider only basis states with odd values of $L$ as these exciton states can be observed in parity-forbidden semiconductors [40, 42, 47].

\section{B. Constant scaled energy}

Besides solving the Schrödinger equation or the generalized eigenvalue problem 10 for fixed values of the external field strength, it is also possible to use the concept of scaled energy [55. In classical mechanics the Hamiltonian of a hydrogen atom in external fields possesses a scaling property which allows reducing the three parameters energy $E$, magnetic field $B$ and electric field $F$ to two parameters [69, 170]. The corresponding transformation reads

$$
\begin{array}{ll}
\hat{\boldsymbol{r}}=\gamma^{2 / 3} \boldsymbol{r}, & \hat{\boldsymbol{p}}=\gamma^{-1 / 3} \boldsymbol{p}, \\
\hat{\boldsymbol{F}}=\gamma^{-4 / 3} \boldsymbol{F}, & \hat{E}=\gamma^{-2 / 3} E,
\end{array}
$$

with $\gamma=B / B_{0}$ and $B_{0}=2.3505 \times 10^{5} \mathrm{~T}[4$. This scaling is not applicable in quantum mechanics since $\left[\hat{r}_{i}, \hat{p}_{j}\right]=$ $i \hbar \gamma^{1 / 3} \delta_{i j} \neq i \hbar \delta_{i j}$ holds. However, it is possible to define a scaled quantum Hamiltonian by substituting $\hat{\boldsymbol{r}}=\gamma^{2 / 3} \boldsymbol{r}$ in the Schrödinger equation and introducing the scaled energy $\hat{\boldsymbol{E}}=\gamma^{-2 / 3} \boldsymbol{E}$.

We will now apply this scaling to the exciton system. Let us write the Hamiltonian of excitons (6) in the form

$$
\begin{aligned}
H & =-\frac{e^{2}}{4 \pi \varepsilon_{0} \varepsilon} \frac{1}{r}-e \boldsymbol{F} \cdot \boldsymbol{r} \\
& +H_{0}+(e B) H_{1}+(e B)^{2} H_{2}
\end{aligned}
$$

with the $H_{i}$ given in Appendix A. Due to the effective masses of electron and hole and due to the scaling of the Coulomb energy by the dielectric constant, we introduce exciton Hartree units so that the hydrogen-like part of the Hamiltonian is exactly of the same form as that of the hydrogen Hamiltonian in normal Hartree units 39 (see Appendix B). Variables in exciton Hartree units will be indicated by a tilde sign.

Performing the substitution $\hat{\boldsymbol{r}}=\gamma^{2 / 3} \tilde{\boldsymbol{r}} / \alpha$ in the corresponding Schrödinger equation, where we now have to use $\gamma=B / B_{0}$ with $B_{0}=2.3505 \times 10^{5} \mathrm{~T} /\left(\gamma_{1}^{\prime 2} \varepsilon^{2}\right)$, and multiplying the resulting equation with $\alpha^{2} \gamma^{2 / 3}$, we ob- tain

$$
\begin{aligned}
& -\frac{\alpha}{\hat{r}}-\gamma^{4 / 3} \alpha^{3} \tilde{\boldsymbol{F}} \cdot \hat{\boldsymbol{r}} \\
+ & \gamma^{2 / 3} \tilde{H}_{0}+\gamma^{1 / 3} \alpha^{2} \tilde{H}_{1}+\alpha^{4} \tilde{H}_{2} \\
= & \gamma^{-2 / 3} \alpha^{2} \tilde{E} .
\end{aligned}
$$

As for the hydrogen atom, we define the scaled energy $\hat{E}=\gamma^{-2 / 3} \tilde{E}$ and scaled electric field strength $\hat{\boldsymbol{F}}=\gamma^{4 / 3} \tilde{\boldsymbol{F}}$. When using the complete basis of Eq. $[9]$, Eq. (14) represents a quadratic eigenvalue problem of the form

$$
\boldsymbol{A c}+\tau \boldsymbol{B} \boldsymbol{c}=\tau^{2} \boldsymbol{C c}
$$

with hermitian matrices $\boldsymbol{A}, \boldsymbol{B}$, and $\boldsymbol{C}$ and an eigenvalue $\tau=\gamma^{1 / 3}$. The eigenvalue problem can be changed to a standard generalized eigenvalue problem by defining a vector $\boldsymbol{d}=\tau \boldsymbol{c}$ :

$$
\left(\begin{array}{cc}
A & B \\
0 & 1
\end{array}\right)\left(\begin{array}{l}
c \\
d
\end{array}\right)=\tau\left(\begin{array}{ll}
0 & C \\
1 & 0
\end{array}\right)\left(\begin{array}{l}
c \\
d
\end{array}\right) .
$$

This eigenvalue problem is solved for constant scaled energies $E$ using an appropriate LAPACK routine [68].

We finally note that due to the substitution $\hat{\boldsymbol{r}}=$ $\gamma^{2 / 3} \tilde{\boldsymbol{r}} / \alpha$ and due to the use of exciton Hartree units, a different value of the free convergence parameter $\alpha$ than in Sec. II has to be used to obtain convergence for the exciton states with principal quantum number $n$. This value is given by $\alpha \approx n \gamma^{2 / 3}$.

\section{DISCUSSION OF ANTIUNITARY SYMMETRIES}

In a previous paper 36] we have shown analytically that the last remaining antiunitary symmetry known from the hydrogen atom in external fields is broken for the exciton Hamiltonian (6) for most orientations of the external fields. For the reader's convenience we recapitulate the most important steps as some of the results are important for the following discussions.

The matrices $\boldsymbol{I}_{i}$ of the quasi-spin $I=1$ given by Eq. (5) are not the standard spin matrices $\boldsymbol{S}_{i}$ of spin one [71. However, these matrices obey the commutation rules [58]

$$
\left[\boldsymbol{I}_{i}, \boldsymbol{I}_{j}\right]=i \hbar \sum_{k=1}^{3} \varepsilon_{i j k} \boldsymbol{I}_{k}
$$

for which reason a unitary transformation can be found so that $\boldsymbol{U}^{\dagger} \boldsymbol{I}_{i} \boldsymbol{U}=\boldsymbol{S}_{i}$ holds. Since in Ref. [7] the behavior of the standard spin matrices under symmetry operations such as time reversal and reflections are given, we will use the matrices $\boldsymbol{S}_{i}$ instead of the $\boldsymbol{I}_{i}$ in the following.

In the special case of vanishing Luttinger parameters $\gamma_{2}=\gamma_{3}=0$, the exciton Hamiltonian (6) is of the same 
form as the Hamiltonian of a hydrogen atom in external fields. It is well known that for this Hamiltonian there is still one antiunitary symmetry left, i.e., that it is invariant under the combined symmetry of time inversion $K$ followed by a reflection $S_{\hat{\boldsymbol{n}}}$ at the specific plane spanned by both fields [7]. This plane is given by the normal vector

$$
\hat{\boldsymbol{n}}=(\boldsymbol{B} \times \boldsymbol{F}) /|\boldsymbol{B} \times \boldsymbol{F}|
$$

or $\hat{\boldsymbol{n}} \perp \hat{\boldsymbol{B}}=\boldsymbol{B} / B$ if $\boldsymbol{F}=\mathbf{0}$ holds. Therefore, the hydrogen-like system shows GOE statistics in the chaotic regime.

As the hydrogen atom is spherically symmetric in the field-free case, it makes no difference whether the magnetic field is oriented in $z$ direction or not. However, in a semiconductor with $\delta^{\prime} \neq 0$ the Hamiltonian has cubic symmetry and the orientation of the external fields with respect to the crystal axis of the lattice becomes important. Any rotation of the coordinate system with the aim of making the $z$ axis coincide with the direction of the magnetic field will also rotate the cubic crystal lattice. The only remaining antiunitary symmetry mentioned above is now broken for the exciton Hamiltonian if the plane spanned by both fields is not identical to one of the symmetry planes of the cubic lattice. Even without an external electric field the symmetry is broken if the magnetic field is not oriented in one of these symmetry planes. Only if the plane spanned by both fields is identical to one of the symmetry planes of the cubic lattice, the antiunitary symmetry $K S_{\hat{\boldsymbol{n}}}$ with $\hat{\boldsymbol{n}}$ given by Eq. (20) is present since only then the reflection $S_{\hat{\boldsymbol{n}}}$ transforms the lattice into itself.

This criterion can also be expressed in a different way: The antiunitary symmetry known from the hydrogen atom is broken if none of the normal vectors $\hat{\boldsymbol{n}}_{i}$ of the 9 symmetry planes of the cubic lattice given by

$$
\begin{aligned}
& \hat{\boldsymbol{n}}_{1}=(1,0,0)^{\mathrm{T}}, \\
& \hat{\boldsymbol{n}}_{2}=(0,1,0)^{\mathrm{T}}, \\
& \hat{\boldsymbol{n}}_{3}=(0,0,1)^{\mathrm{T}}, \\
& \hat{\boldsymbol{n}}_{4}=(1,1,0)^{\mathrm{T}} / \sqrt{2}, \\
& \hat{\boldsymbol{n}}_{5}=(0,1,1)^{\mathrm{T}} / \sqrt{2}, \\
& \hat{\boldsymbol{n}}_{6}=(1,0,1)^{\mathrm{T}} / \sqrt{2}, \\
& \hat{\boldsymbol{n}}_{7}=(1,-1,0)^{\mathrm{T}} / \sqrt{2}, \\
& \hat{\boldsymbol{n}}_{8}=(0,1,-1)^{\mathrm{T}} / \sqrt{2}, \\
& \hat{\boldsymbol{n}}_{9}=(-1,0,1)^{\mathrm{T}} / \sqrt{2},
\end{aligned}
$$

is parallel to

$$
\hat{\boldsymbol{n}}=(\boldsymbol{B} \times \boldsymbol{F}) /|\boldsymbol{B} \times \boldsymbol{F}|,
$$

or, in the case of $\boldsymbol{F}=\mathbf{0}$, if none of these vectors is perpendicular to

$$
\hat{\boldsymbol{B}}=\boldsymbol{B} / B
$$

Since the breaking of all antiunitary symmetries depends on the relative orientation of the external fields to all normal vectors $\hat{\boldsymbol{n}}_{i}$, we can introduce a parameter which is a qualitative measure for the deviation from the cases with antiunitary symmetry:

$$
\sigma=\left[\sum_{i=1}^{9} \frac{|\boldsymbol{B} \times \boldsymbol{F}|^{2}}{\left|\hat{\boldsymbol{n}}_{i} \times(\boldsymbol{B} \times \boldsymbol{F})\right|^{2}}\right]^{-\frac{1}{2}} .
$$

For the special case of $\boldsymbol{F}=\mathbf{0}$ we define

$$
\sigma=\left[\sum_{i=1}^{9}\left(\hat{\boldsymbol{n}}_{i} \cdot \hat{\boldsymbol{B}}\right)^{-2}\right]^{-\frac{1}{2}}
$$

We have $\sigma=0$ for the cases with antiunitary symmetry; and that symmetry is more and more broken with increasing values of $\sigma$.

Under time inversion $K$ and reflections $S_{\hat{\boldsymbol{n}}}$ at a plane perpendicular to a normal vector $\hat{\boldsymbol{n}}$ the vectors of position $\boldsymbol{r}$, momentum $\boldsymbol{p}$ and spin $\boldsymbol{S}$ transform according to 71

$$
\begin{aligned}
K \boldsymbol{r} K^{\dagger} & =\boldsymbol{r}, \\
K \boldsymbol{p} K^{\dagger} & =-\boldsymbol{p}, \\
K \boldsymbol{S} K^{\dagger} & =-\boldsymbol{S},
\end{aligned}
$$

and

$$
\begin{aligned}
S_{\hat{\boldsymbol{n}}} \boldsymbol{r} S_{\hat{\boldsymbol{n}}}^{\dagger} & =\boldsymbol{r}-2 \hat{\boldsymbol{n}}(\hat{\boldsymbol{n}} \cdot \boldsymbol{r}), \\
S_{\hat{\boldsymbol{n}} \boldsymbol{p}} S_{\hat{\boldsymbol{n}}}^{\dagger} & =\boldsymbol{p}-2 \hat{\boldsymbol{n}}(\hat{\boldsymbol{n}} \cdot \boldsymbol{p}), \\
S_{\hat{\boldsymbol{n}}} \boldsymbol{S} S_{\hat{\boldsymbol{n}}}^{\dagger} & =-\boldsymbol{S}+2 \hat{\boldsymbol{n}}(\hat{\boldsymbol{n}} \cdot \boldsymbol{S}) .
\end{aligned}
$$

For all orientations of the external fields the hydrogenlike part of the Hamiltonian (6) is invariant under $K S_{\hat{\boldsymbol{n}}}$ with $\hat{\boldsymbol{n}}$ given by Eq. (20). However, other parts of the Hamiltonian such as $H_{c}=\left(p_{1}^{2} \boldsymbol{S}_{1}^{2}+\right.$ c.p. $)$ [see Eq. (4)] are not invariant if $\sigma \neq 0$ holds. For example, for the case with $\boldsymbol{B}(0,0)$ and $\boldsymbol{F}(\pi / 6, \pi / 2)$, we obtain

$$
\begin{aligned}
& S_{\hat{\boldsymbol{n}}} K H_{c} K^{\dagger} S_{\hat{\boldsymbol{n}}}^{\dagger}-H_{c} \\
= & 1 / 8\left[2 \sqrt{3}\left(\boldsymbol{S}_{2}^{2}-\boldsymbol{S}_{1}^{2}\right) p_{1} p_{2}\right. \\
+ & 3\left(\boldsymbol{S}_{1}^{2} p_{2}^{2}+\boldsymbol{S}_{2}^{2} p_{1}^{2}\right)-3\left(\boldsymbol{S}_{1}^{2} p_{1}^{2}+\boldsymbol{S}_{2}^{2} p_{2}^{2}\right) \\
+ & \left.\left\{\boldsymbol{S}_{1}, \boldsymbol{S}_{2}\right\}\left(2 \sqrt{3}\left(p_{2}^{2}-p_{1}^{2}\right)+12 p_{1} p_{2}\right)\right] \neq 0
\end{aligned}
$$

with $\hat{\boldsymbol{n}}=(-1 / 2, \sqrt{3} / 2,0)^{\mathrm{T}}$. Note that even though $H_{c}$ does not depend on the external fields, the normal vector $\hat{\boldsymbol{n}}$ is determined by these fields via Eq. 20. Otherwise, the hydrogen-like part of the Hamiltonian would not be invariant under $K S_{\hat{\boldsymbol{n}}}$.

Since the expression in Eq. (26) is not equal to zero, we have shown for $\boldsymbol{B}(0,0)$ and $\boldsymbol{F}(\pi / 6, \pi / 2)$ that the generalized time-reversal symmetry of the hydrogen atom is broken for excitons due to the cubic symmetry of the semiconductor. The same calculation can also be performed for other orientations of the external fields. As we have stated above, the antiunitary symmetry remains unbroken only for specific orientations of the fields. 


\section{APPEARANCE OF GOE AND GUE STATISTICS}

We will now demonstrate the breaking of all antiunitary symmetries by analyzing the nearest-neighbor spacings of the energy eigenvalues corresponding to the Hamiltonian (6) 21] for a model system with the arbitrarily chosen set of parameters $E_{\mathrm{g}}=0, \varepsilon=7.5$, $m_{\mathrm{e}}=m_{0}, \gamma_{1}^{\prime}=2, \mu^{\prime}=0$, and $\delta^{\prime}=-0.15$. If we set $\boldsymbol{F}=\mathbf{0}$, we expect to obtain GUE statistics in the limit of high energies as long as the magnetic field is not oriented in one of the symmetry planes of the lattice.

Before analyzing the nearest-neighbor spacings, we have to unfold the spectra to obtain a constant mean spacing [1, 7, 21, 72]. The unfolding procedure separates the average behavior of the non-universal spectral density from universal spectral fluctuations and yields a spectrum in which the mean level spacing is equal to unity $[5$.

To unfold the spectra, we plot for the both cases of constant field strengths and of constant scaled energy the number

$$
N(E)=\sum_{n} \Theta\left(E-E_{n}\right)
$$

of energy levels up to the value $E_{\max }$, up to which all eigenvalues converged. Here $\Theta(x)$ denotes the Heaviside function. We leave out a certain number of low-lying sparse levels to remove individual but nontypical fluctuations [21. In the case of constant scaled energy it is known that the mean number of levels is proportional to $E^{-2 / 3}$ in the dense part of the spectrum 21. Hence, we fit $N(E)$ with $\bar{N}(E)=a E^{-2 / 3}+b$. In the case of constant field strength no such proportionality is known and we fit $N(E)$ with a cubic polynomial function $\bar{N}(E)$. The level spacings of the unfolded spectrum are then given by $s_{n}=\bar{N}\left(E_{n+1}\right)-\bar{N}\left(E_{n}\right)$ 73].

Since the magnetic field breaks all symmetries in the system and limits the convergence of the solutions of the generalized eigenvalue problem with high energies [42, the number of level spacings analyzed here is comparatively small and comprises about 250 to 500 exciton states. In this case, the cumulative distribution function [74]

$$
F(s)=\int_{0}^{s} P(x) \mathrm{d} x
$$

is often more meaningful than histograms of the level spacing probability distribution function $P(s)$.

We will compare our results with the distribution functions known from random matrix theory [1, 34]: the Poissonian distribution

$$
P_{\mathrm{P}}(s)=e^{-s}
$$

for non-interacting energy levels, the Wigner distribution

$$
P_{\mathrm{GOE}}(s)=\frac{\pi}{2} s e^{-\pi s^{2} / 4},
$$

and the distribution

$$
P_{\mathrm{GUE}}(s)=\frac{32}{\pi^{2}} s^{2} e^{-4 s^{2} / \pi}
$$

for systems without any antiunitary symmetry. It can be seen that the most striking difference between the three distributions is the behavior for small values of $s$. While for the Poissonian distribution the probability of level crossings in nonzero and thus $P_{\mathrm{P}}(0) \neq 0$ holds, in chaotic spectra the symmetry reduction leads to a correlation of levels and hence to a strong suppression of crossings. Note that the most characteristic feature of GOE or GUE statistics is the linear or quadratic level repulsion for small $s$, respectively.

In Fig. 1 we show the results for level spacing probability distribution function and the cumulative distribution function for $\boldsymbol{B}(0, \pi / 6)$ and $\boldsymbol{B}(\pi / 6, \pi / 6)$ obtained with a constant magnetic field strength of $B=3 \mathrm{~T}$ and exciton states within a certain energy range. While for $\boldsymbol{B}(0, \pi / 6)$ the magnetic field is oriented in one of the symmetry planes of the lattice and thus only GOE statistics can be observed, we see clear evidence for GUE statistics as regards the case with $\boldsymbol{B}(\pi / 6, \pi / 6)$. Note that we have chosen the values $\delta^{\prime}=-0.15$ and $B=3 \mathrm{~T}$ to be fixed. It is well known from atomic physics that chaotic effects become more apparent in higher magnetic fields or by using states of higher energies for the analysis. Hence, by increasing $B$ or investigating the statistics of exciton states with higher energies, GUE statistics could probably be observed also for smaller values of $\left|\delta^{\prime}\right|$. At this point we have to note that an evaluation of numerical spectra for $\delta^{\prime}>0$ shows the same appearance of GUE statistics. This is expected since the analytically shown breaking of all antiunitary symmetries in Sec. III is independent of the sign of the material parameters.

\section{TRANSITIONS BETWEEN SPACING DISTRIBUTIONS}

To the best of our knowledge, there are only two physical systems where both the transition from Poissonian to GUE statistics and the transition from GOE to GUE statistics in dependence of a parameter of the system could be studied [27, 28. As we have already stated in Secs. III] and IV] our system shows Poisson, GOE or GUE statistics in dependence on the energy, the magnetic field strength and the angles $\vartheta$ and $\varphi$, i.e., in dependence of experimentally adjustable parameters. Thus, our system is perfectly suited to investigate transitions between the different statistics or different symmetry classes when changing one or more of these parameters.

In Ref. [5] analytical expressions for the spacing distribution functions in the transition region between the different statistics have been derived using random matrix theory for $2 \times 2$ matrices. The transition from Poissonian 

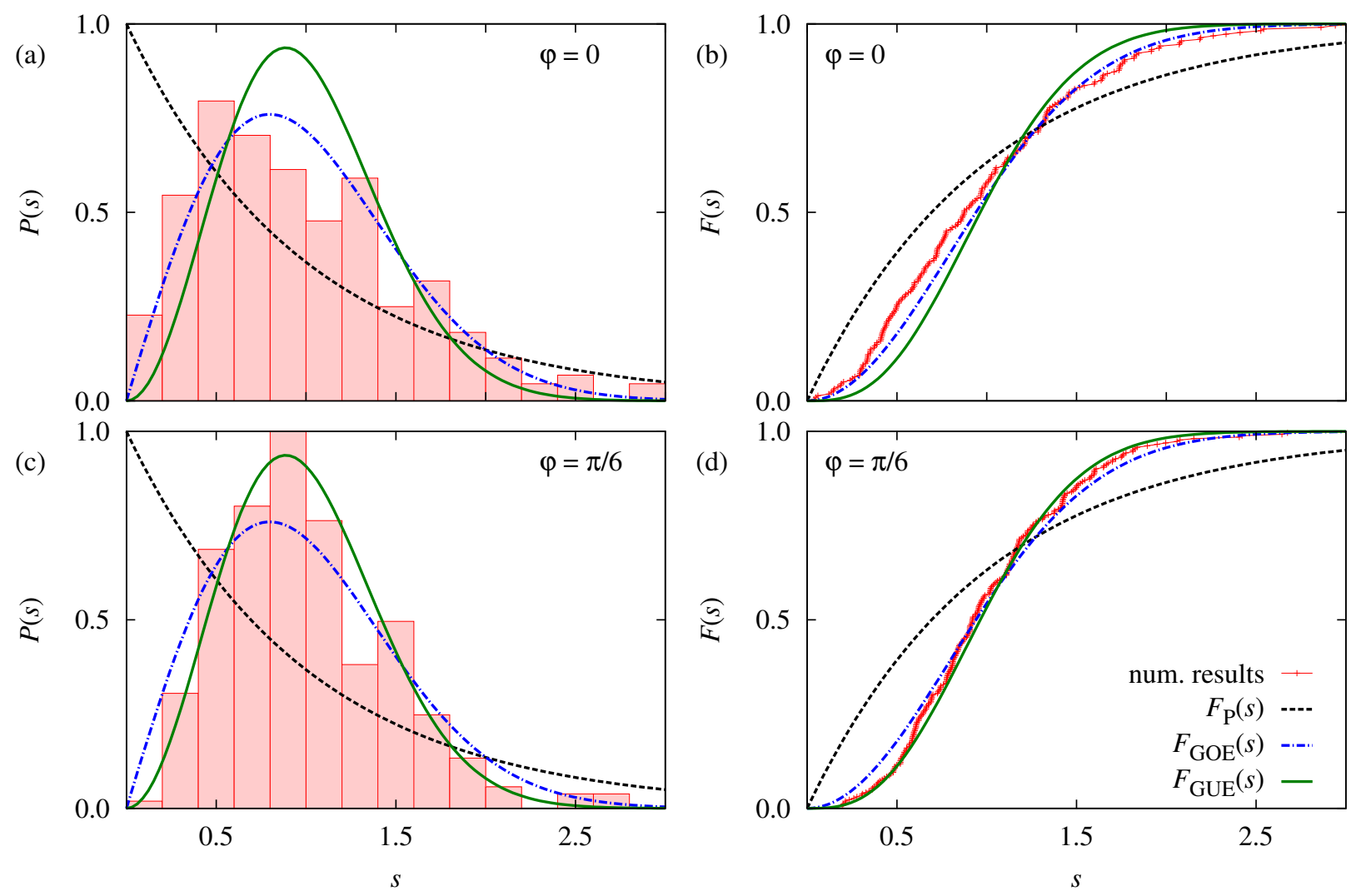

FIG. 1: Level spacing probability distribution functions $P(s)$ (left) and cumulative distribution functions $F(s)$ (right) for $\delta^{\prime}=-0.15, B=3 \mathrm{~T}, \vartheta=\pi / 6$, and two different values of $\varphi$. Besides the numerical data (red boxes or red dots), we also show the corresponding functions of a Poissonian ensemble (black dashed line), GOE (blue dash-dotted line), and GUE (green solid line). Only if the magnetic field is oriented in one of the symmetry planes of the lattice, one antiunitary symmetry is present and GOE statistics can be observed (a,b). In all other cases, all antiunitary symmetries are broken and GUE statistics appears $(\mathrm{c}, \mathrm{d})$.

to GOE statistics is described by

$$
\begin{aligned}
P_{\mathrm{P} \rightarrow \mathrm{GOE}}(s ; \lambda) & =C s e^{-D^{2} s^{2}} \\
& \times \int_{0}^{\infty} \mathrm{d} x e^{-x^{2} / 4 \lambda^{2}-x} I_{0}(z)
\end{aligned}
$$

with $z=x D s / \lambda$ and

$$
\begin{aligned}
& D(\lambda)=\frac{\sqrt{\pi}}{2 \lambda} U\left(-\frac{1}{2}, 0, \lambda^{2}\right), \\
& C(\lambda)=2 D(\lambda)^{2},
\end{aligned}
$$

a parameter $\lambda$, the Tricomi confluent hypergeometric function $U(a, b z)$ 75] and the modified Bessel function $I_{0}(z)$ [75. For the special cases of $\lambda \rightarrow 0$ or $\lambda \rightarrow \infty$ Poissonian or GOE statistics is obtained, respectively. However, already for $\lambda \gtrsim 0.7$ the transition to GOE statistics is almost completed [5].

At this point we have to note that the transition between different symmetry classes is not universal and that the level spacing distributions are universal only in the Poisson, GOE or GUE limit. Besides the transition formula 32 derived within random matrix theory also other interpolating distributions for the transition $\mathrm{P} \rightarrow$ GOE have been proposed in the literature [76 80 . When using one of these distributions for the intermediate regime the results may be modified. However, since all the transition formulae presented here were derived in the same manner within random matrix theory, we use these formula for a consistent description of all transitions considered here.

The transition from Poissonian to GUE statistics is described by

$$
\begin{aligned}
P_{\mathrm{P} \rightarrow \mathrm{GUE}}(s ; \lambda) & =C s^{2} e^{-D^{2} s^{2}} \\
& \times \int_{0}^{\infty} \mathrm{d} x e^{-x^{2} / 4 \lambda^{2}-x} \frac{\sinh (z)}{z}(33 \mathrm{a})
\end{aligned}
$$



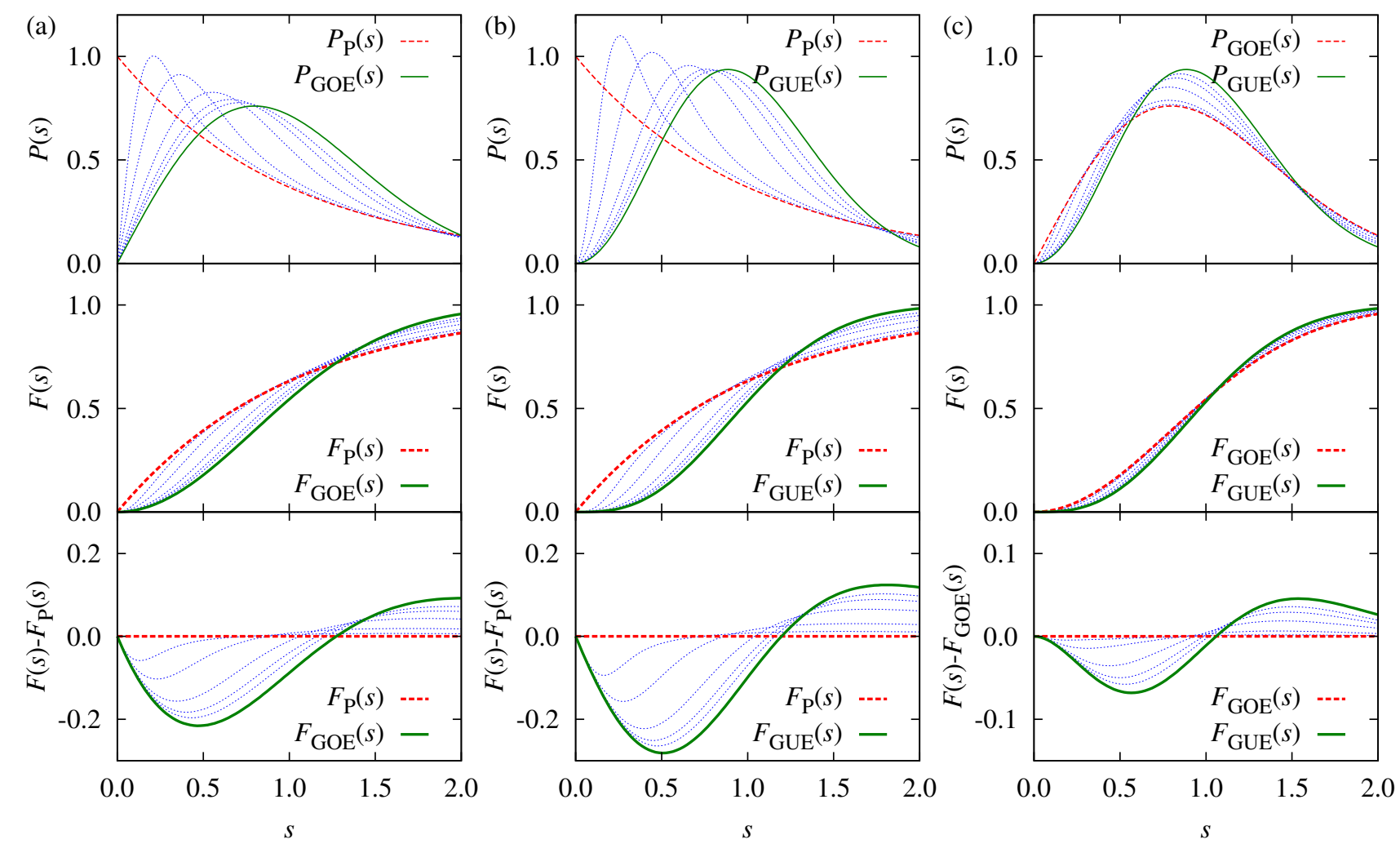

FIG. 2: (Color online) Level spacing probability distribution functions $P(s)$ (first row) and cumulative distribution functions $F(s)$ (second row) for the transitions (a) $\mathrm{P} \rightarrow$ GOE, (b) $\mathrm{P} \rightarrow$ GUE, and (c) GOE $\rightarrow$ GUE. The blue dotted lines show the transition functions of Eqs. (32), (33), and $(34)$ for $\lambda=0.1,0.2,0.4,0.6,0.8$. To visualize the differences between the cumulative distribution functions more clearly, especially for the GOE $\rightarrow$ GUE transition, we also show in the third row the difference between the distributions for a fixed value of $\lambda$ and the initial distribution, respectively.

with $z=x D s / \lambda$ and

$$
\begin{aligned}
D(\lambda) & =\frac{1}{\sqrt{\pi}}+\frac{1}{2 \lambda} e^{\lambda^{2}} \operatorname{erfc}(\lambda)-\frac{\lambda}{2} \operatorname{Ei}\left(\lambda^{2}\right) \\
& +\frac{2 \lambda^{2}}{\sqrt{\pi}}{ }_{2} F_{2}\left(\frac{1}{2}, 1 ; \frac{3}{2}, \frac{3}{2} ; \lambda^{2}\right) \\
C(\lambda) & =\frac{4 D(\lambda)^{3}}{\sqrt{\pi}}
\end{aligned}
$$

the complementary error function erfc [75, the exponential integral Ei [75] and a generalized hypergeometric function ${ }_{2} F_{2}$ [81.

Finally, the transition from GOE to GUE statistics is given by

$$
P_{\mathrm{GOE} \rightarrow \mathrm{GUE}}(s ; \lambda)=C s e^{-D^{2} s^{2}} \operatorname{erf}\left(\frac{D s}{\lambda}\right)
$$

with

$$
\begin{aligned}
& D(\lambda)=\frac{\sqrt{1+\lambda^{2}}}{\sqrt{\pi}}\left(\frac{\lambda}{1+\lambda^{2}}+\operatorname{arccot}(\lambda)\right), \\
& C(\lambda)=2 \sqrt{1+\lambda^{2}} D(\lambda)^{2} .
\end{aligned}
$$

As in Ref. 5, we calculate the distribution functions for $\lambda=0.01 \times 1000^{(k-1) / 999}$ with $k=1, \ldots, 1000$ and then numerically integrate the results to obtain the corresponding cumulative distribution functions $F(s ; \lambda)$. All these functions are shown for different values of $\lambda$ in Fig. 2

As the transition from Poissonian to GOE statistics has been investigated in detail for the hydrogen atom in external fields [21], we will treat the two other transitions in the following.

\section{A. GOE $\rightarrow$ GUE}

Let us start with the transition from GOE to GUE statistics. For this case we solve the generalized eigenvalue problem (10) for different orientations of the magnetic field $\boldsymbol{B}(\varphi, \vartheta)$ by setting $\vartheta=\pi / 6$ and gradually increasing the angle $\varphi$ from 0 to $\pi / 4$. To increase the statistical significance, we analyze and merge the level spacings for $B=2.8 \mathrm{~T}, B=3.0 \mathrm{~T}$, and $B=3.2 \mathrm{~T}$ for a given value of $\varphi$ [21]. The results are finally fitted by the 

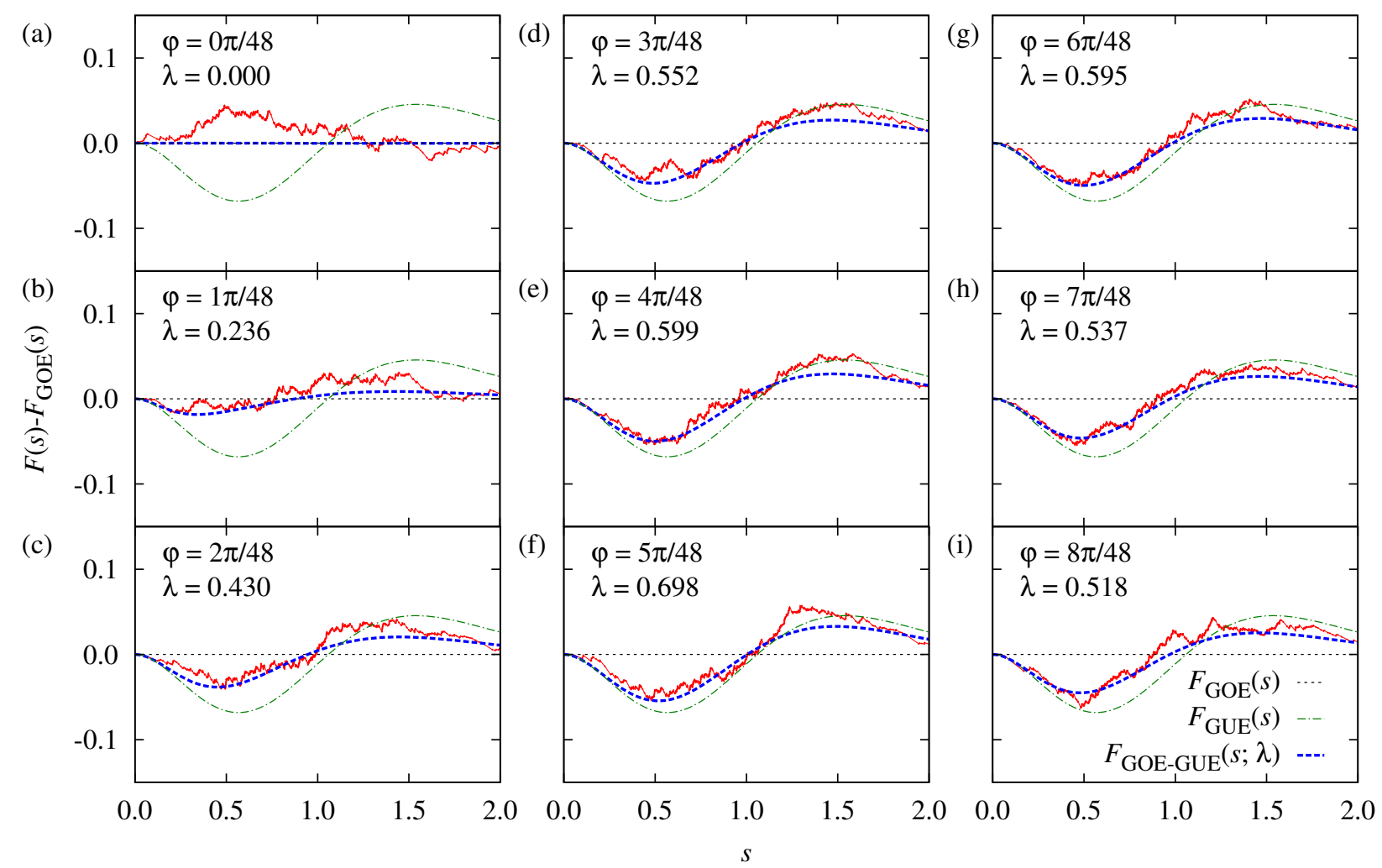

FIG. 3: Transition from GOE to GUE statistics for fixed values of the magnetic field strength $B$ and increasing values of the angle $\varphi$ in $\boldsymbol{B}(\varphi, \vartheta=\pi / 6)$. The results are presented in the same way as in the bottom most panel of Fig. 2 to show the differences between $F_{\mathrm{GOE}}(s)$ and $F_{\mathrm{GUE}}(s)$ more clearly. The data points (red) were fitted with the analytical function $F_{\mathrm{GOE} \rightarrow \mathrm{GUE}}(s ; \lambda)$. The optimum values of the fit parameter $\lambda$ are given in each panel, but also shown in Fig. 4 . One can observe a good agreement between the numerical data and the analytical function describing the transition between the two statistics in dependence on $\lambda$. Only for $\varphi=0$ the data shows a slight admixture of Poissonian statistics to the expected GOE statistics. For further information see text.
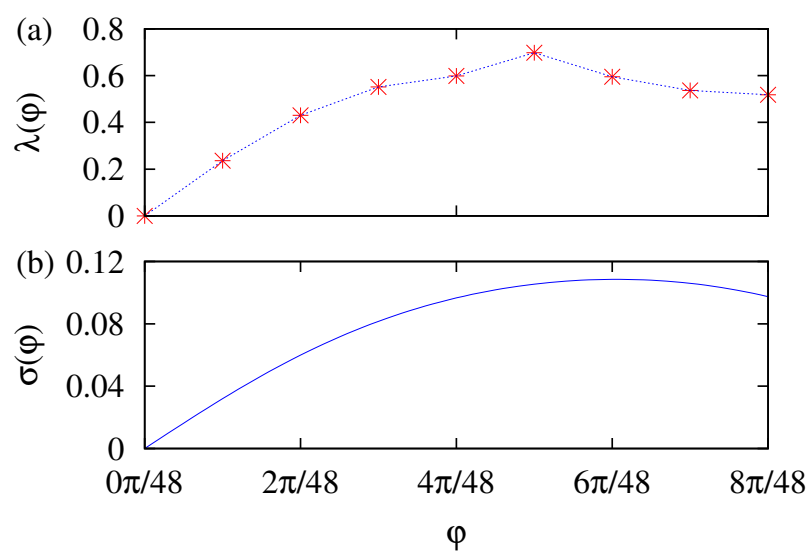

FIG. 4: (a) Optimum values of the fit parameter $\lambda$ in dependence on the angle $\varphi$ for the situation presented in Fig. 3. The blue dashed line only serves as a guide to the eye. (b) The function $\sigma(\varphi)$ of Eq. 23) for $\vartheta=\pi / 6$. We obtain a qualitatively good agreement between both curves, i.e., as expected, both values $\lambda(\varphi)$ and $\sigma(\varphi)$ increase from zero to a certain value and then decrease for $\varphi \gtrsim \pi / 8$. function $F_{\mathrm{GOE} \rightarrow \mathrm{GUE}}(s ; \lambda)$ and shown in Fig. 3.

For the special case of $\varphi=0$ we obtain GOE statistics as expected since the magnetic field is oriented in the symmetry plane of the solid with $\hat{\boldsymbol{n}}=(0,1,0)^{\mathrm{T}}$. When increasing the angle $\varphi$, the parameter $\lambda$ changes rapidly from 0 to 0.5 and hence the transition from GOE to GUE statistics is almost completed for $\varphi \gtrsim 3 \pi / 48$ (see Fig. 4).

The decrease of the parameter $\lambda$ for $\varphi \gtrsim \pi / 8$ in Fig. 4 can be explained by considering the orientation of $\boldsymbol{B}$ with respect to all symmetry planes of the lattice. Hence, we calculate the value of the parameter $\sigma$ of Eq. (23) for $\vartheta=\pi / 6$ and increasing values of $\varphi$. It is obvious that the value of $\sigma$ increases for $0 \leq \varphi \leq \pi / 8$ and decreases for $\pi / 8 \leq \varphi \leq \pi / 4$ since the magnetic field moves away from the plane with $\hat{\boldsymbol{n}}_{2}$ and then approaches the plane with $\hat{\boldsymbol{n}}_{7}$. Therefore, the fact that $\boldsymbol{B}$ approaches the plane with $\hat{\boldsymbol{n}}_{7}$ for $\varphi \geq \pi / 8$ explains the decrease of $\lambda$ in Fig. 3 . 

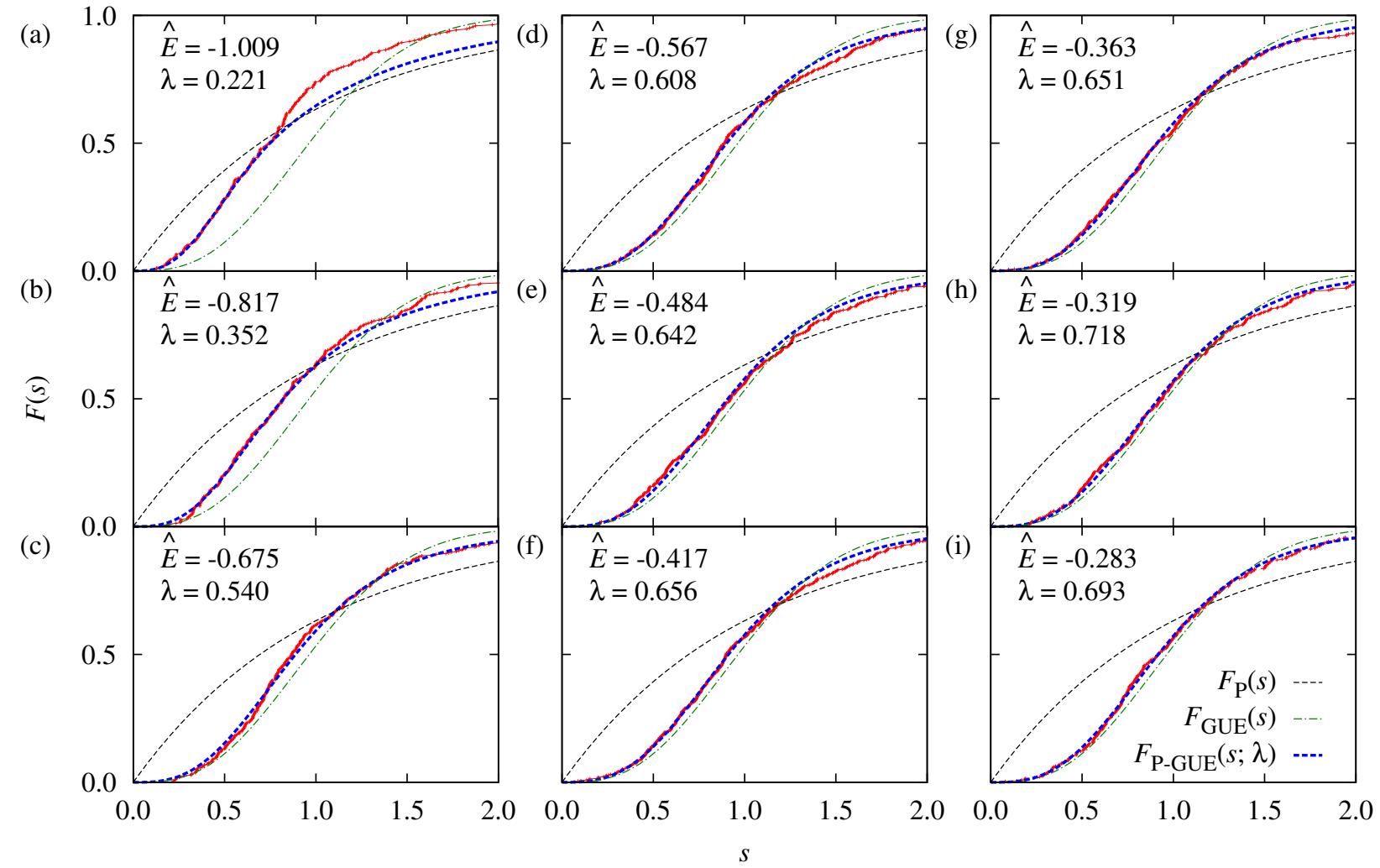

FIG. 5: Transition from Poissonian to GUE statistics for fixed values of the angles $\varphi=\pi / 8, \vartheta=\pi / 6$ and increasing values of the scaled energy $\hat{E}$. Except for $\hat{E}=-1.009$ a good agreement between the numerical data and the analytical function $F_{\mathrm{P} \rightarrow \mathrm{GUE}}(s ; \lambda)$ is obtained. For further information see text.

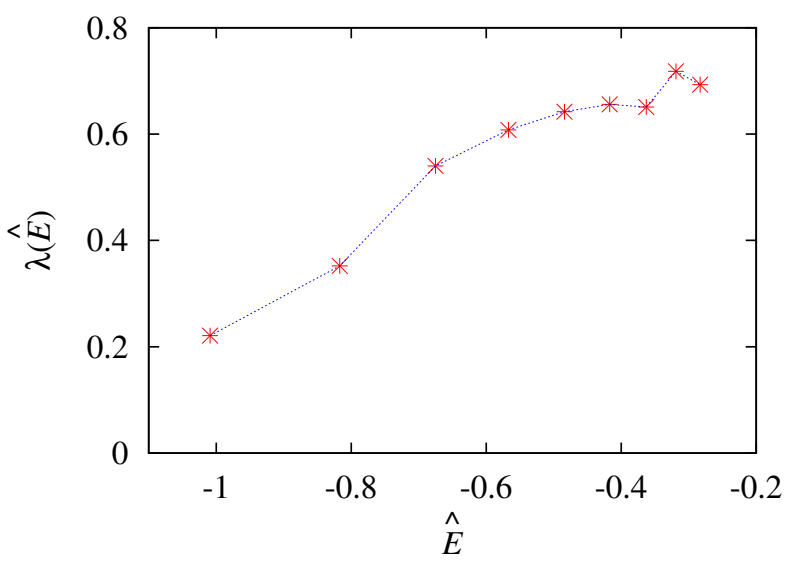

FIG. 6: Optimum values of the fit parameter $\lambda$ in dependence on the scaled energy $\hat{E}$ for the situation presented in Fig. 5 The blue dashed line only serves as a guide to the eye. The value of $\lambda$ increases from a small value at low scaled energies to about $\lambda \approx 0.7$, where the function $F_{\mathrm{P} \rightarrow \mathrm{GUE}}(s ; \lambda)$ almost describes GUE statistics.

\section{B. Poisson $\rightarrow$ GUE}

Let us now treat the transition from Poissonian to GUE statistics. It is known from the hydrogen atom in external fields that for fixed values of the magnetic field strength $B$ the low-energy part of the eigenvalue spectrum will show Poissonian statistics while the highenergy part already shows GOE statistics. For a better level statistics it is appropriate to analyze the spectra with a constant scaled energy $\hat{E}$.

For fixed small values of the scaled energy the corresponding classical dynamics becomes regular and energy eigenvalues of the quantum mechanical system will show purely Poissonian statistics. On the other hand, as we have shown above, GUE statistics is observed best at large energies and for angles $\varphi$ and $\vartheta$, for which the magnetic field is oriented exactly between two symmetry planes of the lattice. Hence, keeping the values $\varphi=\pi / 8$, $\vartheta=\pi / 6$, and $\delta^{\prime}=-0.15$ fixed and increasing the scaled energy, we expect to observe a transition from Poissonian to GUE statistics.

Having unfolded the spectra according to Ref. [21], we fit the numerical results by the function $F_{\mathrm{P} \rightarrow \mathrm{GUE}}(s ; \lambda)$ given in Eq. (33). It can be seen from Fig. 5 that we obtain a good agreement between the results for our sys- 
tem and the analytical function for all scaled energies $\hat{E}>-0.9$. The transition from Poissonian to GUE statistics takes place already at very small values of the scaled energy $-1.2 \lesssim \hat{E} \lesssim-0.6$ (see Fig. 6). This differs from the hydrogen atom in external fields where the statistics is still Poisson-like for $\hat{E} \lesssim-0.6$ [21] and can be explained by the presence of the cubic band structure here. Therefore, the presence of the cubic band structure increases the chaos in comparison with the hydrogen atom.

For very small values of the scaled energy $\hat{E} \lesssim-0.8$ a reasonable analysis of the spectra is hardly possible. For these values of $\hat{E}$ we cannot obtain enough converged eigenvalues in the dense part of the spectrum due to the required computer memory. On the other hand, the number of low-lying sparse levels increases. Hence, fitting the number $N(E)$ of energy levels with the function $\bar{N}(E)=a E^{-2 / 3}+b$ for the unfolding procedure (cf. Sec. IV) does not lead to good results since the mean number of energy levels is proportional to $E^{-2 / 3}$ only in the dense part of the spectrum. This effect can already be observed for $\hat{E}=-1.009$ in Fig. 5 . Note that a change in the unfolding procedure or the fit function would not lead to better results as the problem is connected with the appearance of the low-lying sparse levels. These levels lead to individual but nontypical fluctuations [21].

It is generally assumed that the NNS of large random matrices can be approximated by the NNS of $2 \times 2$ matrices of the same universality class [5]. Since we obtained a good agreement when fitting the functions $F_{\mathrm{GOE} \rightarrow \mathrm{GUE}}(s ; \lambda)$ and $F_{\mathrm{P} \rightarrow \mathrm{GUE}}(s ; \lambda)$, which were derived for $2 \times 2$ matrices, to our numerical results, we could prove the Wigner surmise [56] for our system.

\section{SUMMARY AND OUTLOOK}

Investigating the Hamiltonian of excitons in cubic semiconductors we could show analytically and numerically that the simultaneous presence of the cubic band structure and external fields can break all antiunitary symmetries in the system. The level spacing statistics of the quantum mechanical spectrum depends on the energy, the field strengths, the field orientations and on the value of the parameter $\delta^{\prime}$, which determines the strength of the cubic deformation of the band structure. This makes excitons in external fields a prime system to investigate the transitions between different level spacing statistics. Keeping the parameter $\delta^{\prime}$ fixed, we analyzed the transition from GOE to GUE statistics and from Poissonian to GUE statistics. A comparison with analytical formulae for these transitions derived for $2 \times 2$ matrices within random matrix theory showed very good agreements. Hence, we could confirm the Wigner surmise for our model system.

Since we changed only parameters such as the angles of the magnetic field or the scaled energy, which can also be varied in experiments, we think that the transition between the different level statistics could also be in- vestigated experimentally. However, changing the two parameters $\delta^{\prime}$ and the scaled energy $\hat{E}$ in numerical calculations will allow us to investigate arbitrary transitions of the level statistics in the triangle between Poissonian (arbitrary $\delta^{\prime}$, small $\left.\hat{E}\right)$, GOE $\left(\delta^{\prime}=0\right.$, large $\left.\hat{E}\right)$, and GUE statistics $\left(\delta^{\prime} \neq 0\right.$, large $\left.\hat{E}\right)$ in the future. As for arbitrary transitions within this triangle no analytical formulae have been derived within random matrix theory so far, the corresponding functions $P\left(s ; \lambda_{1}, \lambda_{2}\right)$ also have to be found.

We want to note that all transitions considered here are modelled by Hamiltonians of the form $H=H_{\beta}+\lambda H_{\beta^{\prime}}$ [5], where $H_{\beta^{\prime}}$ has a lower symmetry than $H_{\beta}$. The level statistics is strongly affected by the perturbation $H_{\beta^{\prime}}$ if the level spacings of $H_{\beta}$, which are smaller than the matrix elements of this Hamiltonian, and the matrix elements of $\lambda H_{\beta^{\prime}}$ are of comparable size. In the case of $\hbar \rightarrow 0$, the transition will take place at even smaller values of $\lambda$. Especially, the connection between $\lambda$ and the parameter $\sigma$ [cf. Eqs. (22) and [23)] must depend on $\hbar$. However, we note that the parameter $\sigma$ has only been introduced phenomenologically to describe the dependency of the transition on the angle between the vector $\hat{\boldsymbol{n}} 20$ or $\hat{\boldsymbol{B}} 21$ and the normal vectors $\hat{\boldsymbol{n}}_{i}$ of the symmetry planes of the lattice.

To investigate the dependence of all results on $\hbar$, further and more extensive calculations are necessary, which is beyond the scope of this work. Nevertheless, our model system offers the possibility for an according analysis and we will discuss the effects in a future publication.

Finally, we are certain that the discovery of GUE statistics for giant Rydberg excitons may pave the way to a deeper understanding of the connection between quantum and classical chaos.

\section{Acknowledgments}

F.S. is grateful for support from the Landesgraduiertenförderung of the Land Baden-Württemberg. We thank D. Fröhlich, M. Aßmann and M. Bayer for helpful discussions.

\section{Appendix A: Hamiltonian}

In this section we give the complete Hamiltonian of Eq. (6) and describe the rotation necessary to make the quantization axis coincide with the direction of the magnetic field. Let us write the Hamiltonian (6) in the form

$$
\begin{aligned}
H & =E_{\mathrm{g}}-\frac{e^{2}}{4 \pi \varepsilon_{0} \varepsilon} \frac{1}{r} \\
& +H_{0}+(e B) H_{1}+(e B)^{2} H_{2}-e \boldsymbol{F} \cdot \boldsymbol{r}
\end{aligned}
$$

with $B=|\boldsymbol{B}|$. Using $\hat{B}_{i}=B_{i} / B$ with the components $B_{i}$ of $\boldsymbol{B}$, the terms $H_{0}, H_{1}$, and $H_{2}$ are given by 
TABLE I: Exciton Hartree units converted to SI-units for $\gamma_{1}^{\prime}=2$ and $\varepsilon=7.5$. For a comparison, we also give the values for normal Hartree units, which are obtained by setting $\gamma_{1}^{\prime}=\varepsilon=1$.

\begin{tabular}{lllll}
\hline quantity & symbol & exc. Hartree unit & SI $\left(\gamma_{1}^{\prime}=2, \varepsilon=7.5\right)$ & SI $\left(\gamma_{1}^{\prime}=1, \varepsilon=1\right)$ \\
\hline \hline charge & $q$ & $e$ & $1.6022 \times 10^{-19} \mathrm{C}$ & $1.6022 \times 10^{-19} \mathrm{C}$ \\
action & $S$ & $\hbar$ & $1.0546 \times 10^{-34} \mathrm{Js}$ & $1.0546 \times 10^{-34} \mathrm{Js}$ \\
mass & $m$ & $m_{0} / \gamma_{1}^{\prime}$ & $4.5547 \times 10^{-31} \mathrm{~kg}$ & $9.1094 \times 10^{-31} \mathrm{~kg}$ \\
length & $r$ & $\gamma_{1}^{\prime} \varepsilon a_{0}$ & $7.9377 \times 10^{-10} \mathrm{~m}$ & $5.2918 \times 10^{-11} \mathrm{~m}$ \\
momentum & $p$ & $\hbar / \gamma_{1}^{\prime} \varepsilon a_{0}$ & $1.3286 \times 10^{-25} \mathrm{~kg} \mathrm{~m} / \mathrm{s}$ & $1.9929 \times 10^{-24} \mathrm{~kg} \mathrm{~m} / \mathrm{s}$ \\
time & $t$ & $\gamma_{1}^{\prime} \varepsilon^{2} a_{0}^{2} m_{0} / \hbar$ & $2.7213 \times 10^{-15} \mathrm{~s}$ & $2.4189 \times 10^{-17} \mathrm{~s}$ \\
energy & $E$ & $\hbar^{2} / \gamma_{1}^{\prime} \varepsilon^{2} a_{0}^{2} m_{0}$ & $3.8753 \times 10^{-20} \mathrm{~J}$ & $4.3597 \times 10^{-18} \mathrm{~J}$ \\
magn. flux density & $B$ & $\hbar / \gamma_{1}^{\prime 2} \varepsilon^{2} a_{0}^{2} e$ & $1.0447 \times 10^{+3} \mathrm{~T}$ & $2.3505 \times 10^{+5} \mathrm{~T}$ \\
el. field strength & $F$ & $\hbar^{2} / \gamma_{1}^{\prime 2} \varepsilon^{3} a_{0}^{3} m_{0} e$ & $3.0472 \times 10^{+8} \mathrm{~V} / \mathrm{m}$ & $5.1422 \times 10^{+11} \mathrm{~V} / \mathrm{m}$ \\
\hline
\end{tabular}

$$
\begin{aligned}
H_{0} & =\frac{1}{2 m_{0}}\left(\gamma_{1}^{\prime}+4 \gamma_{2}\right) \boldsymbol{p}^{2}-\frac{3 \gamma_{2}}{\hbar^{2} m_{0}}\left[\boldsymbol{I}_{1}^{2} p_{1}^{2}+\text { c.p. }\right] \\
& -\frac{6 \gamma_{3}}{\hbar^{2} m_{0}}\left[\left\{\boldsymbol{I}_{1}, \boldsymbol{I}_{2}\right\} p_{1} p_{2}+\text { c.p. }\right]
\end{aligned}
$$

$$
\begin{aligned}
& H_{1}= \frac{1}{2 m_{0}}\left(\frac{2 m_{0}}{m_{\mathrm{e}}}-\gamma_{1}^{\prime}+4 \gamma_{2}\right) \hat{\boldsymbol{B}} \cdot \boldsymbol{L} \\
&+ \frac{3 \gamma_{2}}{\hbar^{2} m_{0}}\left[\boldsymbol{I}_{1}^{2}\left(\hat{B}_{2} r_{3} p_{1}-\hat{B}_{3} r_{2} p_{1}\right)+\text { c.p. }\right] \\
&+\frac{3 \gamma_{3}}{\hbar^{2} m_{0}}\left[\{ \boldsymbol { I } _ { 1 } , \boldsymbol { I } _ { 2 } \} \left(\hat{B}_{2} r_{3} p_{2}-\hat{B}_{1} r_{3} p_{1}\right.\right. \\
&\left.\left.+\hat{B}_{3} r_{1} p_{1}-\hat{B}_{3} r_{2} p_{2}\right)+ \text { c.p. }\right]
\end{aligned}
$$$$
H_{2}=\frac{1}{8 m_{0}}\left(\gamma_{1}^{\prime}+4 \gamma_{2}\right)\left[\hat{\boldsymbol{B}}^{2} \boldsymbol{r}^{2}-(\hat{\boldsymbol{B}} \cdot \boldsymbol{r})^{2}\right]
$$$$
-\frac{3 \gamma_{2}}{4 \hbar^{2} m_{0}}\left[\boldsymbol{I}_{1}^{2}\left(\hat{B}_{2} r_{3}-\hat{B}_{3} r_{2}\right)^{2}+\text { c.p. }\right]
$$$$
-\frac{3 \gamma_{3}}{2 \hbar^{2} m_{0}}\left[\left\{\boldsymbol{I}_{1}, \boldsymbol{I}_{2}\right\}\left(\hat{B}_{2} r_{3}-\hat{B}_{3} r_{2}\right)\right.
$$$$
\left.\times\left(\hat{B}_{3} r_{1}-\hat{B}_{1} r_{3}\right)+\text { c.p. }\right] .
$$

i.e., we replace $\boldsymbol{x} \rightarrow \boldsymbol{x}^{\prime}=\boldsymbol{R}^{\mathrm{T}} \boldsymbol{x}$ with $\boldsymbol{x} \in\{\boldsymbol{r}, \boldsymbol{p}, \boldsymbol{L}, \boldsymbol{I}, \boldsymbol{S}\}$ to make the quantization axis coincide with the direction of the magnetic field [65, 66. Finally we express the Hamiltonian in terms of irreducible tensors (see, e.g., Refs. [42, 43, 59, 66]) and calculate the matrix elements of the matrices $\boldsymbol{D}$ and $\boldsymbol{M}$ in the generalized eigenvalue problem 110 or the matrices $\boldsymbol{A}, \boldsymbol{B}$, and $\boldsymbol{C}$ in the generalized eigenvalue problem (15).

\section{Appendix B: Exciton Hartree units}

In our calculations, we express the magnetic field in spherical coordinates [see Eq. (7)]. For the different orientations of the magnetic field we rotate the coordinate system by

$$
\boldsymbol{R}=\left(\begin{array}{ccc}
\cos \varphi \cos \vartheta & \sin \varphi \cos \vartheta & -\sin \vartheta \\
-\sin \varphi & \cos \varphi & 0 \\
\cos \varphi \sin \vartheta & \sin \varphi \sin \vartheta & \cos \vartheta
\end{array}\right)
$$

When performing numerical calculations for the hydrogen atom in external fields, often Hartree units are used [39, 82. These units are obtained by setting the fundamental physical constants $e, m_{0}, \hbar$ as well as the Bohr radius $a_{0}$ to one. As the effective masses of the electron and hole differ from the free electron mass and since the Coulomb interaction is scaled by the dielectric constant $\varepsilon$, we introduce exciton Hartree units. Within these units the hydrogen-like part of the Hamiltonian (6) is exactly of the same form as the Hamiltonian of the hydrogen atom in Hartree units 39 and the values of the scaled energies in Sec. IIB can be compared directly with the values of the scaled energies used in calculations for the hydrogen atom 21. The exciton Hartree units are obtained by setting $e=\hbar=1, m_{0}=\gamma_{1}^{\prime}$ and $a_{\text {exc }}=\gamma_{1}^{\prime} \varepsilon a_{0}=1$. Since all other physical quantities have to be converted to exciton Hartree units as well, we give the according scaling factors in Table I. Variables given in exciton Hartree units are marked by a tilde sign, e.g., $r \rightarrow \tilde{r}$, throughout the paper.

[1] O. Bohigas, M. J. Giannoni, and C. Schmit, Phys. Rev. Lett. 52, 1 (1984).

[2] M. L. Mehta, Random Matrices (Elsevier, Amsterdam, 
2004), 3rd ed.

[3] C. E. Porter, ed., Statistical Theory of Spectra (Academic Press, New York, 1965).

[4] J. Rao and K. T. Taylor, J. Phys. B: At. Mol. Opt. Phys. 35, 2627 (2002).

[5] S. Schierenberg, F. Bruckmann, and T. Wettig, Phys. Rev. E 85, 061130 (2012).

[6] G. Lenz and F. Haake, Phys. Rev. Lett. 67, 1 (1991).

[7] F. Haake, Quantum Signatures of Chaos, Springer Series in Synergetics (Springer, Heidelberg, 2010), 3rd ed.

[8] A. Pandey, Ann. Phys. 119, 170 (1979).

[9] G. E. Mitchell, A. Richter, and H. A. Weidenmüller, Rev. Mod. Phys. 82, 2845 (2010).

[10] T. A. Brody, J. Flores, J. B. French, P. A. Mello, A. Pandey, and S. S. M. Wong, Rev. Mod. Phys. 53, 385 (1981).

[11] N. Rosenzweig and C. E. Porter, Phys. Rev. 120, 1698 (1960).

[12] H. S. Camarda and P. D. Georgopulos, Phys. Rev. Lett. 50, 492 (1983).

[13] H.-J. Stöckmann and J. Stein, Phys. Rev. Lett. 64, 2215 (1990).

[14] H. Alt, H.-D. Gräf, H. L. Harney, R. Hofferbert, H. Lengeler, A. Richter, P. Schardt, and H. A. Weidenmüller, Phys. Rev. Lett. 74, 62 (1995).

[15] H. Alt, H.-D. Gräf, R. Hofferbert, C. Rangacharyulu, H. Rehfeld, A. Richter, P. Schardt, and A. Wirzba, Phys. Rev. E 54, 2303 (1996).

[16] T. Zimmermann, H. Köppel, L. S. Cederbaum, G. Persch, and W. Demtröder, Phys. Rev. Lett. 61, 3 (1988).

[17] W. Zhou, Z. Chen, B. Zhang, C. H. Yu, W. Lu, and S. C. Shen, Phys. Rev. Lett. 105, 024101 (2010).

[18] L. Vina, M. Potemski, and W. Wang, Phys.-Usp. 41, 153 (1998).

[19] H. Held, J. Schlichter, G. Raithel, and H. Walther, Europhys. Lett. 43, 392 (1998).

[20] A. Frisch, M. Mark, K. Aikawa, F. Ferlaino, J. L. Bohn, C. Makrides, A. Petrov, and S. Kotochigova, Nature 507, 475 (2014).

[21] D. Wintgen and H. Friedrich, Phys. Rev. A 35, 1464(R) (1987).

[22] H. Friedrich and D. Wintgen, Phys. Rep. 183, 37 (1989).

[23] M. V. Berry and M. Tabor, Proc. Roy. Soc. London A 356, 375 (1977).

[24] P. So, S. M. Anlage, E. Ott, and R. N. Oerter, Phys. Rev. Lett. 74, 2662 (1995).

[25] K. Sacha, J. Zakrzewski, and D. Delande, Phys. Rev. Lett. 83, 2922 (1999).

[26] P. Shukla and A. Pandey, Nonlinearity 10, 979 (1997).

[27] H. Haake, M. Kuś, and R. Scharf, Z. Phys. B 65, 381 (1987).

[28] P. Shukla, J. Phys. Condens. Matter 17, 1653 (2005).

[29] F. J. Dyson, J. Math. Phys. 3, 1191 (1962).

[30] U. Stoffregen, J. Stein, H.-J. Stöckmann, M. Kuś, and F. Haake, Phys. Rev. Lett. 74, 2666 (1995).

[31] L. A. Ponomarenko, F. Schedin, M. I. Katsnelson, R. Yang, E. W. Hill, K. S. Novoselov, and A. K. Geim, Science 320, 356 (2008).

[32] S.-H. Chung, A. Gokirmak, D.-H. Wu, J. S. A. Bridgewater, E. Ott, T. M. Antonsen, and S. M. Anlage, Phys. Rev. Lett. 85, 2482 (2000).

[33] T. Kazimierczuk, D. Fröhlich, S. Scheel, H. Stolz, and M. Bayer, Nature 514, 343 (2014).

[34] M. Aßmann, J. Thewes, D. Fröhlich, and M. Bayer, Na- ture Mater. 15, 741 (2016).

[35] M. Freitag, J. Heckötter, M. Bayer, and M. Aßmann, Phys. Rev. B 95, 155204 (2017).

[36] F. Schweiner, J. Main, and G. Wunner, Phys. Rev. Lett. 118, 046401 (2017).

[37] F. Schweiner, J. Main, and G. Wunner, Phys. Rev. B 93, 085203 (2016).

[38] P. Grünwald, M. Aßmann, J. Heckötter, D. Fröhlich, M. Bayer, H. Stolz, and S. Scheel, Phys. Rev. Lett. 117, 133003 (2016).

[39] M. Feldmaier, J. Main, F. Schweiner, H. Cartarius, and G. Wunner, J. Phys. B: At. Mol. Opt. Phys. 49, 144002 (2016).

[40] J. Thewes, J. Heckötter, T. Kazimierczuk, M. Aßmann, D. Fröhlich, M. Bayer, M. A. Semina, and M. M. Glazov, Phys. Rev. Lett. 115, 027402 (2015), and Supplementary Material.

[41] F. Schöne, S. O. Krüger, P. Grünwald, H. Stolz, S. Scheel, M. Aßmann, J. Heckötter, J. Thewes, D. Fröhlich, and M. Bayer, Phys. Rev. B 93, 075203 (2016).

[42] F. Schweiner, J. Main, M. Feldmaier, G. Wunner, and Ch. Uihlein, Phys. Rev. B 93, 195203 (2016).

[43] F. Schweiner, J. Main, G. Wunner, M. Freitag, J. Heckötter, Ch. Uihlein, M. Aßmann, D. Fröhlich, and M. Bayer, Phys. Rev. B 95, 035202 (2017).

[44] J. Heckötter, M. Freitag, D. Fröhlich, M. Aßmann, M. Bayer, M. A. Semina, and M. M. Glazov, Phys. Rev. B 95, 035210 (2017).

[45] S. Zielińska-Raczyńska, D. Ziemkiewicz, and G. Czajkowski, Phys. Rev. B 95, 075204 (2017).

[46] F. Schweiner, J. Main, G. Wunner, and Ch. Uihlein, Phys. Rev. B 94, 115201 (2016).

[47] S. Zielińska-Raczyńska, G. Czajkowski, and D. Ziemkiewicz, Phys. Rev. B 93, 075206 (2016).

[48] S. Zielińska-Raczyńska, D. Ziemkiewicz, and G. Czajkowski, Phys. Rev. B 94, 045205 (2016).

[49] F. Schweiner, J. Main, G. Wunner, and Ch. Uihlein, Phys. Rev. B 95, 195201 (2017).

[50] C. Klingshirn, Semiconductor Optics (Springer, Berlin, 2007), 3rd ed.

[51] U. Rössler, Solid State Theory (Springer, Berlin, 2009), 2nd ed.

[52] R. Knox, Theory of excitons, vol. 5 of Solid State Physics Supplement (Academic, New York, 1963).

[53] Ch. Uihlein, D. Fröhlich, and R. Kenklies, Phys. Rev. B 23, 2731 (1981).

[54] G. M. Kavoulakis, Y.-C. Chang, and G. Baym, Phys. Rev. B 55, 7593 (1997).

[55] D. Wintgen, Phys. Rev. Lett. 58, 1589 (1987).

[56] E. P. Wigner, in Conference on neutron physics by timeof-flight, edited by R. C. Block, W. M. Good, J. A. Harvey, H. W. Schmitt, and G. T. Trammell (Union Carbide Nuclear Company, Oak Ridge, Tennessee, 1957), vol. 2309 of Oak Ridge National Laboratory Report, pp. 59-70.

[57] N. O. Lipari and M. Altarelli, Phys. Rev. B 15, 4883 (1977).

[58] J. Luttinger, Phys. Rev. 102, 1030 (1956).

[59] A. Baldereschi and N. O. Lipari, Phys. Rev. B 8, 2697 (1973).

[60] P. Schmelcher and L. S. Cederbaum, Z. Phys. D 24, 311 (1992).

[61] P. Schmelcher and L. S. Cederbaum, Phys. Rev. A 47, 2634 (1993). 
[62] M. Altarelli and N. O. Lipari, Phys. Rev. B 7, 3798 (1973).

[63] M. Altarelli and N. O. Lipari, Phys. Rev. B 9, 1733 (1974).

[64] Y. Chen, B. Gil, H. Mathieu, and J. P. Lascaray, Phys. Rev. B 36, 1510 (1987).

[65] J. Broeckx, Phys. Rev. B 43, 9643 (1991).

[66] A. Edmonds, Angular momentum in quantum mechanics (Princeton University Press, Princeton, 1960).

[67] M. A. Caprio, P. Maris, and J. P. Vary, Phys. Rev. C 86, $034312(2012)$.

[68] E. Anderson, Z. Bai, C. Bischof, S. Blackford, J. Demmel, J. Dongarra, J. D. Croz, A. Greenbaum, S. Hammarling, A. McKenney, et al., LAPACK Users' Guide (Society for Industrial and Applied Mathematics, Philadelphia, PA, 1999), 3rd ed.

[69] H. Harada and H. Hasegawa, J. Phys. A 16, L259 (1983).

[70] H. Hasegawa, S. Adachi, and A. Harada, J. Phys. A: Math. Gen. 16, L503 (1983).

[71] A. Messiah, Quantum Mechanics 2 (North-Holland, Amsterdam, 1969).

[72] T. A. Brody, J. Flores, J. B. French, P. A. Mello,
A. Pandey, and S. S. M. Wong, Rev. Mod. Phys. 53, 385 (1981).

[73] S. Keppeler, Spinning Particles - Semiclassics and Spectral Statistics (Springer, Berlin, 2003).

[74] J.-B. Grosa, O. Legranda, F. Mortessagnea, E. Richalotb, and K. Selemanib, Wave Motion 51, 664 (2014).

[75] M. Abramowitz and I. A. Stegun, Handbook of Mathematical Functions (Dover Publ., New York, 1964).

[76] M. V. Berry and M. Robnik, J. Phys. A 17, 2413 (1984).

[77] T. A. Brody, Lett. Nuovo Cimento 7, 482 (1973).

[78] E. Caurier, B. Grammaticos, and A. Ramani, J. Phys. A 23, 4903 (1990).

[79] H. Hasegawa, H. J. Mikeska, and H. Frahm, Phys. Rev. A 38, 395 (1988).

[80] F. Izrailev, Phys. Rep. 5-6, 299 (1990).

[81] I. Gradshteyn and I. Ryzhik, Tables of Integrals, Series, and Products (Academic Press, Burlington, MA, 2007), 7th ed.

[82] P. J. Mohr and B. N. Taylor, Rev. Mod. Phys. 77, 1 (2005). 